\title{
Giant enhancement of the transverse magneto-optical Kerr effect through the coupling of $\varepsilon$-near-zero and surface plasmon polariton modes
}

\author{
J. A. Girón-Sedas, ${ }^{1}$ F. Reyes Gómez, ${ }^{1}$ Pablo Albella, ${ }^{2,3}$ J. R. Mejía-Salazar, ${ }^{4,}{ }^{*}$ and Osvaldo N. Oliveira Jr. ${ }^{4}$ \\ ${ }^{1}$ Departamento de Física, Universidad del Valle, AA 25360, Cali, Colombia \\ ${ }^{2}$ University Institute for Intelligent Systems and Numerical Applications in Engineering (SIANI), \\ University of Las Palmas de Gran Canaria, 35017 Las Palmas de Gran Canaria, Spain \\ ${ }^{3}$ The Blackett Laboratory, Department of Physics, Imperial College London, London SW7 2AZ, United Kingdom \\ ${ }^{4}$ Instituto de Física de São Carlos, Universidade de São Paulo, CP 369, 13560-970 São Carlos, São Paulo, Brazil
}

(Received 14 March 2017; revised manuscript received 13 July 2017; published 11 August 2017)

\begin{abstract}
We demonstrate a concept for the giant enhancement of the transverse magneto-optical Kerr effect (TMOKE), with amplitudes reaching the maximum theoretical values of \pm 1 . The concept exploits the strong electromagnetic field localization in $\varepsilon$-near-zero metamaterials to excite surface plasmon resonances with no need of a prism or grating coupler, thus opening routes for magneto-optical devices amenable to miniaturization. A demonstration of the capability of the enhancement mechanism is presented in which giant TMOKE values can be used for sensing and biosensing.
\end{abstract}

DOI: 10.1103/PhysRevB.96.075415

\section{INTRODUCTION}

The proper control of Kerr and Faraday magneto-optical (MO) effects [1-12] is essential for magnetic storage, biosensing, and optical isolation devices [13-17], in addition to ultrafast control of magnetization via light in magnetic storage systems requiring high operation rates [18-20]. The transverse magneto-optical Kerr effect (TMOKE), in particular, has been useful for biosensing [15,17], optical filtering [16], and magnetization monitoring in magnetic samples $[13,14]$. TMOKE is typically of the order of $10^{-3}$ in conventional ferromagnetic materials [21], but it can be increased considerably by exploiting nanometer-sized structures [1-6]. In principle, this enhancement can be obtained through excitation of surface plasmon polaritons (SPPs) in the conventional Kretschmann geometry, but this would be rather difficult [22] because the refractive index of the prism must be larger than the refractive index of the dielectric background. Moreover, miniaturization is impaired owing to the requirement of a prism for SPP excitation. Although grating coupler platforms [6] allow for miniaturization, we should note that surface plasmon resonance (SPR) excitation depends on the diffraction order, ridge geometry, and the period length of the system, which requires a complex design. The excitation of SPP resonances without a prism or grating coupler has been achieved experimentally [23] by controlling light absorption in nanostructured indium tin oxide (ITO) films operating in the $\varepsilon$-near-zero (ENZ) frequency regime [24-26]. Such excitation was reached through the coupling of the perfect absorbing ENZ mode [27] at the ENZ slab with the allowed SPP mode at the ENZ/metal interface for $p$-polarized incident light.

In this work, we demonstrate theoretically that the direct coupling to SPP can be further exploited as a concept to obtain a giant enhancement of TMOKE, with amplitudes reaching the maximum theoretical values of \pm 1 . The concept is based on the use of a MO ENZ structure for magnetic modulation of SPP resonances while simultaneously maintaining a high level of absorbance. We show that it is possible to obtain the giant enhancement of TMOKE by using a hypothetical

\footnotetext{
*jrmejia3146@gmail.com
}

MO ENZ slab grown over a gold substrate. The vanishing behavior in the dielectric permittivity of the hypothetical slab, responsible for the giant enhancement of TMOKE, is carefully selected to occur at the near-infrared region. This choice was made because we propose later on in this paper a feasible structure with an ITO slab as the ENZ material that can show this effect experimentally. ITO films can be built [28-30] with thicknesses ranging from $\sim 4$ to $\sim 600 \mathrm{~nm}$ to have electron densities in between that of noble metals $\left(10^{22} \mathrm{~cm}^{-3}\right)$ and doped semiconductors $\left(10^{19} \mathrm{~cm}^{-3}\right)$. Also, significant tuning of the electron density of ITO films can be made with electrical or optical methods [31], allowing its use in near-infrared optical modulators and sensing devices [32,33].

TMOKE is a magneto-optical intensity effect, only observable when obliquely incident $p$-polarized light impinges on a system magnetized perpendicular to the incidence plane. It is characterized by the relative change in the reflected light amplitude when the magnetization of the system or an external magnetic field, $\mathbf{M}$, is reversed [21]:

$$
\mathrm{TMOKE}=\frac{R_{\mathrm{pp}}(+\mathbf{M})-R_{\mathrm{pp}}(-\mathbf{M})}{R_{\mathrm{pp}}(+\mathbf{M})+R_{\mathrm{pp}}(-\mathbf{M})},
$$

where $R_{\mathrm{pp}}(+\mathbf{M})$ and $R_{\mathrm{pp}}(-\mathbf{M})$ denote the reflectivity for magnetization along the $\hat{\mathbf{e}}_{y}$ and $-\hat{\mathbf{e}}_{y}$ directions, respectively. Numerical results for TMOKE and $R_{\mathrm{pp}}$ are obtained by using the scattering matrix method [34-37] (SMM), with excellent agreement with experimental results in periodically patterned [36] and Kretschmann-configuration [37] MO multilayer systems.

\section{THEORETICAL FRAMEWORK}

Let us first discuss the case of a hypothetical MO ENZ slab with thickness $l$ and dielectric permittivity tensor given by

$$
\begin{aligned}
& \varepsilon_{x x}=\varepsilon_{y y}=\varepsilon_{\|}=5.53-\frac{v_{\mathrm{p}}^{2}}{v^{2}+i \gamma_{0} v}, \\
& \varepsilon_{z z}=\varepsilon_{\perp}=\frac{53.0 v^{2}+53.0 i \gamma_{0} v-13.25 v_{\mathrm{p}}^{2}}{13.84 v^{2}+13.84 i \gamma_{0} v-1.25 v_{\mathrm{p}}^{2}}, \\
& \varepsilon_{x z}=-\varepsilon_{z x}=0.012 i,
\end{aligned}
$$


where $v_{\mathrm{p}}=0.477 \mathrm{PHz}$ and $\gamma_{0}=4.775 \times 10^{-3} \mathrm{PHz}$, with subindices $\|$ and $\perp$ indicating components parallel and normal to the $x y$ plane. The incidence plane is the $x z$ plane, and the magnetization of the MO ENZ slab is considered normal to the growth direction, $z$ axis, and parallel to the $y$ axis. Around the frequency $v_{\perp}=0.2385 \mathrm{PHz}, \varepsilon_{\perp} \approx 0$, since at this frequency there is a transition from negative to positive values for the real part of permittivity, i.e., $\operatorname{Re}\left\{\varepsilon_{\perp}\left(\nu_{\perp}\right)\right\}=0$. The parameters in Eqs. (2)-(4) were chosen as realistic values for existing materials, as will be discussed later on. In this manuscript, we use a fixed working wavelength at the near infrared corresponding to $\lambda_{0}=1250 \mathrm{~nm}$.

For simplicity, in the analytical treatment, we define $\widehat{\eta}=\widehat{\varepsilon}^{-1}$. Thus,

$$
\widehat{\eta}=\left(\begin{array}{ccc}
\eta_{x x} & 0 & \eta_{x z} \\
0 & \eta_{y y} & 0 \\
\eta_{z x} & 0 & \eta_{z z}
\end{array}\right)
$$

with

$$
\widehat{\eta}=\left(\varepsilon_{\|} \varepsilon_{\perp}+\varepsilon_{x z}^{2}\right)^{-1}\left(\begin{array}{ccc}
\varepsilon_{\perp} & 0 & -\varepsilon_{x z} \\
0 & \left(\varepsilon_{\|} \varepsilon_{\perp}+\varepsilon_{x z}^{2}\right) / \varepsilon_{\|} & 0 \\
\varepsilon_{x z} & 0 & \varepsilon_{\|}
\end{array}\right)
$$

from which we calculate the eigenvector corresponding to $p$-polarized incident light, with $\omega=2 \pi \nu$, as

$$
q_{p}=\sqrt{\frac{\omega^{2}}{c^{2}} \frac{1}{\eta_{x x}}-k_{x}^{2} \frac{\eta_{z z}}{\eta_{x x}}} .
$$

For the incident medium or gold substrate, we use $q_{i}=\sqrt{\frac{\omega^{2}}{c^{2}} \frac{1}{\eta_{i}}-k_{x}^{2}}$, with $i$ being 0 or Au, respectively. From now on, we use $q_{\mathrm{ENZ}}=q_{p}$ for simplicity in discussing the results. The conditions for the excitation of perfectly absorbing (PA) ENZ modes and SPP resonances, for the system in Fig. 1, can be obtained from SMM for anisotropic media $[35,36]$ as

$$
e^{2 i q_{\mathrm{ENZ}} d}=\frac{\left(q_{0} \eta_{0}-q_{\mathrm{ENZ}} \eta_{x x}+k_{x} \eta_{x z}\right)\left(q_{\mathrm{Au}} \eta_{\mathrm{Au}}+q_{\mathrm{ENZ}} \eta_{x x}+k_{x} \eta_{x z}\right)}{\left(q_{0} \eta_{0}+q_{\mathrm{ENZ}} \eta_{x x}+k_{x} \eta_{x z}\right)\left(q_{\mathrm{Au}} \eta_{\mathrm{Au}}-q_{\mathrm{ENZ}} \eta_{x x}+k_{x} \eta_{x z}\right)}
$$

and

$$
\tan \left(q_{\mathrm{ENZ}} d\right)=\frac{-i q_{\mathrm{ENZ}}\left(q_{0} \eta_{0}+q_{\mathrm{Au}} \eta_{\mathrm{Au}}\right) \eta_{x x}}{q_{\mathrm{Au}} q_{0} \eta_{0} \eta_{\mathrm{Au}}+\left(q_{0} \eta_{0}-q_{\mathrm{Au}} \eta_{\mathrm{Au}}\right) k_{x} \eta_{x z}+\left(q_{\mathrm{ENZ}} \eta_{x x}\right)^{2}-\left(k_{x} \eta_{x z}\right)^{2}},
$$

respectively, where $q_{0}, q_{\mathrm{Au}}$, and $q_{\mathrm{ENZ}}$ correspond to the wave vectors in air, gold, and ENZ slab. $k_{\mathrm{ENZ}}$ and $k_{\mathrm{SPP}}$ are obtained by solving $k_{x}$ from the above equations for each case.

\section{RESULTS AND DISCUSSION}

Figure 1 depicts the system under study with its permittivity $(\varepsilon)$ and reflectivity $\left(R_{\mathrm{pp}}\right)$ as functions of the frequency and angle of incidence, $\theta$, around the ENZ region. The corresponding dispersion relations $\operatorname{Re}\left\{k_{\mathrm{ENZ}}\right\}, k_{0}=\omega / c$, and $\operatorname{Re}\left\{k_{\mathrm{SPP}}\right\}$ are also plotted. The absorbance is usually defined as $A=1-R-T$, where $R$ and $T$ correspond to the reflectance and transmittance, respectively. In the present case, the absorbance becomes $A=1-R$ as the ENZ slab is backed by a metallic substrate, thus yielding an effectively zero transmittance. A range of $\theta$ values with negligible reflectance, i.e., very high absorbance, is observed in Fig. 1(c) within the ENZ region. Such behavior can be explained in terms of the strong electromagnetic field localization due to the excitation of the ENZ mode in the ENZ slab [24-26]. According to the dispersion relations in Fig. 1(d), SPP modes can be directly excited by the incident light without a prism or grating coupler [23].

Figure 2(a) shows that giant TMOKE values can only be obtained when PA ENZ modes are very close to or match the SPP resonances, i.e., when a coupling of ENZ-SPP modes occurs. Strongly enhanced TMOKE values appear only when strong absorption is linked to the excitation of SPP resonances, as indicated in Figs. 3(a)-3(d). It is significant that reflectance values of the order of $10^{-4}$ have been measured in related experiments [38], but measuring lower values as obtained in our work may be challenging. The coupling of ENZ modes to other types of modes has been demonstrated; for example, the strong optical coupling in an ENZ material-semiconductor hybrid structure is suitable for a highly tunable integrated device [39]. For small thicknesses, TMOKE can have a preferential sign, while for larger values, it has the usual Fano-like resonance shape, as seen in Fig. 2(b).

An experimentally feasible system to observe giant TMOKE values due to coupling of PA ENZ with SPP modes is depicted in Fig. 4(a). The platform is designed as a trilayer arrangement of ITO/BIG-YIG/SiO 2 grown over a gold substrate, which can be developed with available experimental techniques $[40,41]$. In this platform, the ITO slab is responsible for the ENZ behavior, while the BIG:YIG slab contributes with $\mathrm{MO}$ effects and $\mathrm{SiO}_{2}$ and $\mathrm{Au}$ serve as the substrate. The ITO layer is described by an isotropic permittivity $\varepsilon_{\text {ITO }}=\varepsilon_{\infty}-\frac{v_{\mathrm{p}}^{2}}{v^{2}+i \gamma v}$, where $\gamma=0.03 \mathrm{PHz}$ and $v_{\mathrm{p}}=0.477 \mathrm{PHz}$ denote the damping rate and plasma frequency, respectively. We used $\varepsilon_{\infty}=4, m^{*}=0.4 m_{e}$, and $N=1.13 \times 10^{21} \mathrm{~cm}^{-3}$ for the high-frequency permittivity, effective mass, and carrier density, respectively, taken from experimental values in Ref. [23]. Parameters for BIG-YIG were taken from Refs. [7,12]. The TMOKE curves obtained as a function of $\theta$ in Fig. 4(b) change significantly when the refractive index $(\mathrm{RI})$ of the incident medium is varied, which occurs even for very small changes. Figure 4(c) shows that $\theta$ for the maximum amplitude of TMOKE in Fig. 4(b) varies linearly with the change in RI, with a slope $S=-25^{\circ} /$ RIU. Since ITO films may be functionalized with phosphonates [42], amines [43], zirconium complexes [44], carboxylic acids and thiols [45], DNA [46], and silanes [47], the results in Fig. 4 can be exploited, for example, in designing novel sensing/biosensing 

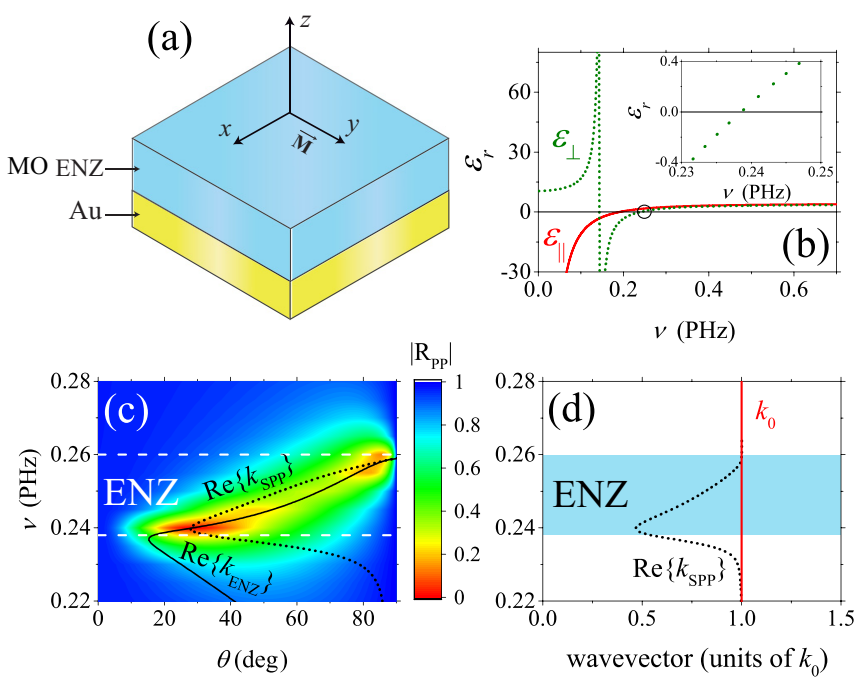

FIG. 1. (a) Schematic representation of the system under study. (b) Permittivities parallel and normal to the plane of magnetization for the hypothetical ENZ slab. The inset shows a zoom of the permittivity around their zero value. (c) Reflectance as a function of frequency and incident angle $\theta$, calculated by using the SMM. Horizontal dashed lines are used to depict the ENZ region. Solid and dotted lines correspond to the numerical solutions for perfect absorbing modes $\left(k_{\mathrm{ENZ}}\right)$ and SPPs at the ENZ/gold interface $\left(k_{\mathrm{SPP}}\right)$ in order to highlight the regions where a matching of both modes can occur in this system. (d) Numerical solutions for the real part of the wave vectors, along the $x$ axis, in air $\left(k_{0}\right)$ and SPPs at the ENZ/gold interface. The MO ENZ slab thickness was taken as $l=0.09 \lambda \mathrm{nm}$, where $\lambda$ is the incident wavelength.

platforms to detect or monitor very small RI changes in the incident medium, with high precision. Significantly, the $S$ value is of the same order of magnitude as in a recent Kretschmann-based SPR sensor for detecting glucose [48]. Figure $4(\mathrm{~d})$ shows the TMOKE, from -0.1 to 0.1 (for visual purposes), as a function of the incident medium dielectric permittivity $\varepsilon_{0}$ and incident angle $\theta$ from which a change of sign is clearly observed. A qualitative interpretation of this sign alternation can be given by considering the trilayer system as just one effective medium (similar to the basic case shown in Fig. 1) to calculate the corresponding SPR and ENZ modes shown by the dashed and solid lines in Fig. 4(d), respectively.
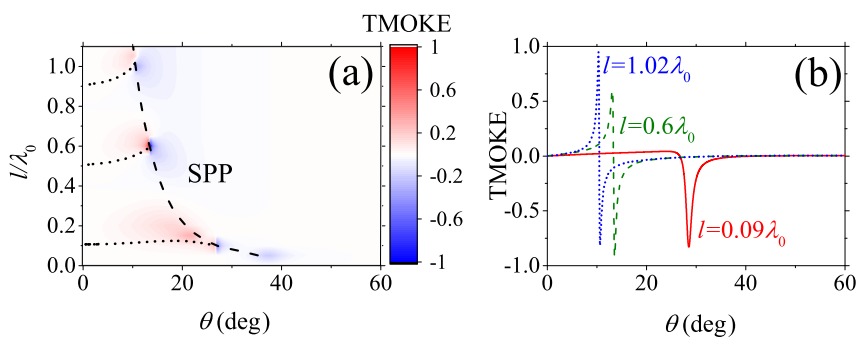

FIG. 2. (a) TMOKE as a function of the angle of incidence and thickness, $l / \lambda_{0}$, of the MO ENZ slab for a working wavelength $\lambda_{0}=$ $1250 \mathrm{~nm}$. The dotted and dashed lines indicate the perfectly absorbing (PA) ENZ and SPP modes, from Eqs. (8) and (9), respectively. (b) TMOKE for three thicknesses of the effective MO ENZ slab.
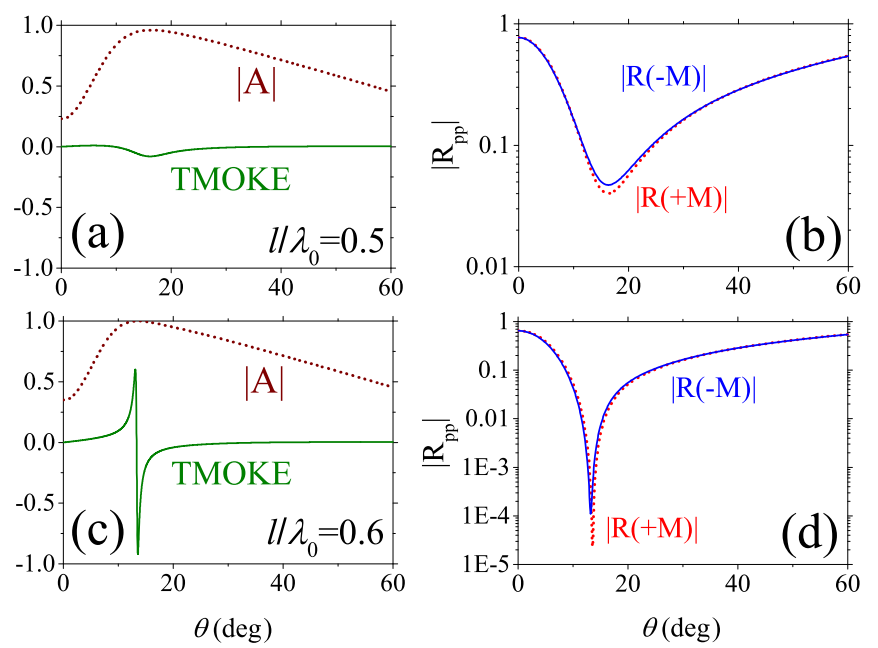

FIG. 3. (a),(b) and (c),(d) show the absorption, TMOKE, $R_{p p}(+\mathbf{M})$, and $R_{p p}(-\mathbf{M})$, for $l / \lambda_{0}=0.5$ and $l / \lambda_{0}=0.6$, respectively. The dotted and solid lines in (a) and (c) correspond to the absorption and TMOKE, respectively. High absorbances with low TMOKE values are observed for $l / \lambda_{0}=0.5$, in comparison with the corresponding PA ENZ solution in Fig. 2(a) in the main text, while giant TMOKE values are obtained at the intersection of PA ENZ and SPP modes for $l / \lambda_{0}=0.6$.

The TMOKE amplitude is again noted to be preferentially positive/negative in the quasimatching of PA ENZ and SPP modes, while an oscillatory behavior should be observed in the matching condition. This actually further confirms that the ENZ-SPP coupling is responsible for the enhanced TMOKE. The effective medium approximation was made by considering
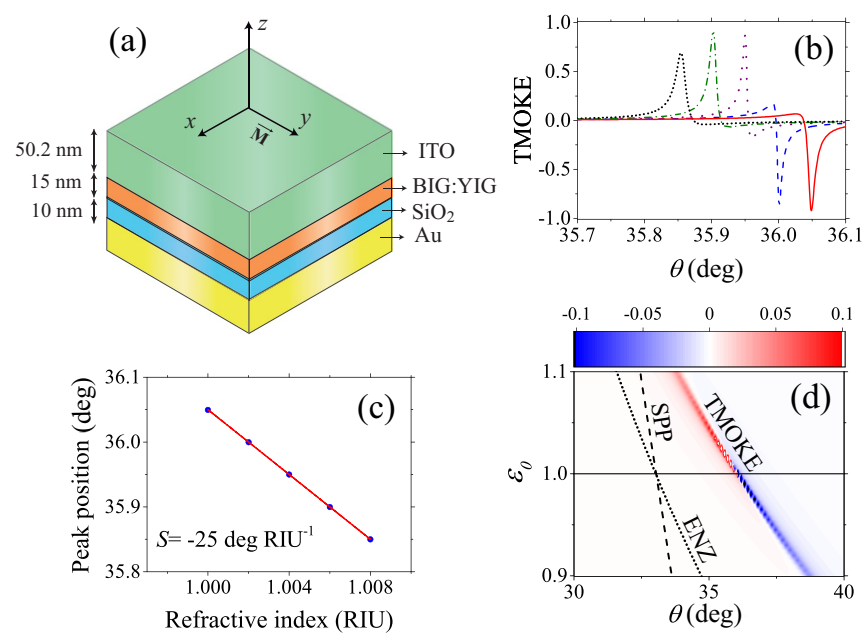

FIG. 4. Pictorial view of the multilayer system on a gold substrate. (b) TMOKE as a function of the incident angle for five values of RI. Solid, dashed, dotted, dash-dotted, and dot-dotted lines are for RI values of 1.000, 1.002, 1.004, 1.006, and 1.008, respectively. (c) TMOKE peak position as a function of RI, showing a linear behavior with slope $S=-25 \mathrm{deg} \mathrm{RIU}^{-1}$. (d) TMOKE as a function of the dielectric permittivity of the incident medium, $\varepsilon_{0}$, and angle of incidence. The dashed and dotted lines show the solutions for SPP and ENZ modes when the system is considered as an effective medium. 

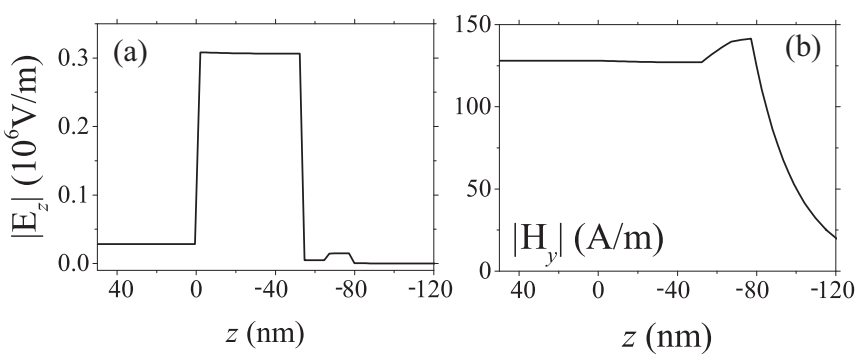

FIG. 5. (a) Electric $E_{z}$ and (b) magnetic $H_{y}$ fields along the multilayer structure for light impinging with an incident angle $\theta=36^{\circ}$. In (a), $E_{z}$ is strongly localized at the ITO layer. The net enhancement of TMOKE is caused by the strong interaction of the localized electromagnetic field at the ENZ layer with adjacent MO bismuth iron garnet:yttrium iron garnet (BIG:YIG) layer. Note also the localization of $H_{y}$ at the dielectric/metal interface in (b), thus showing the excitation of SPP mode.

that the thickness of each layer satisfies the subwavelength condition [49] $d_{\mathrm{SiO}_{2}}, d_{\mathrm{MO}}, d_{\mathrm{ITO}} \lesssim \lambda_{0} / 10$, where $d_{\mathrm{SiO}_{2}}, d_{\mathrm{MO}}, d_{\mathrm{ITO}}$ are the thicknesses of the $\mathrm{SiO}_{2}$, BIG:YIG, and ITO layers. Thus, we have used [50-54]

$$
\begin{aligned}
\varepsilon_{\|} & =f_{\mathrm{ITO}} \varepsilon_{\mathrm{ITO}}+f_{\mathrm{MO}} \varepsilon_{\mathrm{MO}}+f_{\mathrm{SiO}_{2}} \varepsilon_{\mathrm{SiO}_{2}}, \\
\varepsilon_{\perp} & =\frac{\varepsilon_{\mathrm{ITO}} \varepsilon_{\mathrm{MO}} \varepsilon_{\mathrm{SiO}_{2}}}{f_{\mathrm{SiO}_{2}} \varepsilon_{\mathrm{ITO}} \varepsilon_{\mathrm{MO}}+f_{\mathrm{MO}} \varepsilon_{\mathrm{ITO}} \varepsilon_{\mathrm{SiO}_{2}}+f_{\mathrm{ITO}} \varepsilon_{\mathrm{MO}} \varepsilon_{\mathrm{SiO}_{2}}}, \\
\varepsilon_{x z} & =f_{\mathrm{MO}} \varepsilon_{x z, \mathrm{MO}},
\end{aligned}
$$

in Eqs. (6)-(9), where subindices ITO, $\mathrm{MO}$, and $\mathrm{SiO}_{2}$ denote the parameters corresponding to ITO, BIG-YIG, and $\mathrm{SiO}_{2}$ materials, respectively. The filling ratios of $\mathrm{SiO}_{2}$, BIG-YIG, and ITO can be defined as $f_{\mathrm{SiO}_{2}}=\frac{d_{\mathrm{SiO}_{2}}}{d}, f_{\mathrm{MO}}=\frac{d_{\mathrm{MO}}}{d}$, and $f_{\text {ITO }}=1.0-f_{\mathrm{SiO}_{2}}-f_{\mathrm{MO}}$, with $d$ being the total thickness of the structure, $d=d_{\mathrm{ITO}}+d_{\mathrm{SiO}_{2}}+d_{\mathrm{MO}}$. One should note that
Eqs. (2)-(4) were obtained by fixing all parameters according to Fig. 4.

The mechanism responsible for the high sensitivity in the architecture in Fig. 4(a) can be further supported by the corresponding magnitudes for the electric and magnetic field profiles, $\left|E_{z}\right|$ and $\left|H_{y}\right|$, along the structure. Figure 5(a) shows a localized electric field strongly enhanced in the ITO film, thus leading to enhanced MO interactions with the adjacent BIGYIG layer. Strongly enhanced MO phenomena are produced, while the magnetic field is enhanced at the dielectric/metal interface, as seen in Fig. 5(b), thus corroborating the excitation of a SPP resonance.

\section{CONCLUSIONS}

In summary, we have shown that the coupling of perfectly absorbing ENZ modes with SPP resonances in magnetophotonic structures enhances TMOKE up to giant values. In some cases, TMOKE could reach the maximum theoretical amplitudes of \pm 1 , to be compared with the typical $10^{-3}$ in conventional ferromagnetic materials. The concepts introduced open up the possibility for the design and development of highly integrated nanometer-sized magnetoplasmonic devices. By way of illustration, we described the case of a realistic multilayer architecture that can be used as a sensing/biosensing platform with proper functionalization.

\section{ACKNOWLEDGMENTS}

We acknowledge the financial support from the Colombian agency COLCIENCIAS and the Brazilian agencies CNPq and FAPESP (Grants No. 2013/14262-7 and No. 2016/12311-9). P.A. acknowledges Programa "Viera y Clavijo" de la Agencia Canaria de Investigación, Innovación y Sociedad de la Información (ACIISI) y la Universidad de las Palmas de Gran Canaria (ULPGC).
[1] V. I. Belotelov, D. A. Bykov, L. L. Doskolovich, A. N. Kalish, and A. K. Zvezdin, J. Opt. Soc. Am. B 26, 1594 (2009).

[2] V. I. Belotelov, D. A. Bykov, L. L. Doskolovich, A. N. Kalish, and A. K. Zvezdin, J. Exp. Theor. Phys. 110, 816 (2010).

[3] A. A. Grunin, A. G. Zhdanov, A. A. Ezhov, E. A. Ganshina, and A. A. Fedyanin, Appl. Phys. Lett. 97, 261908 (2011).

[4] V. I. Belotelov, I. A. Akimov, M. Pohl, V. A. Kotov, S. Kasture, A. S. Vengurlekar, A. V. Gopal, D. R. Yakovlev, A. K. Zvezdin, and M. Bayer, Nat. Nanotech. 6, 370 (2011).

[5] M. Pohl, L. E. Kreilkamp, V. I. Belotelov, I. A. Akimov, A. N. Kalish, N. E. Khokhlov, V. J. Yallapragada, A. V. Gopal, M. Nur-E-Alam, M. Vasiliev, D. R. Yakovlev, K. Alameh, A. K. Zvezdin, and M. Bayer, New J. Phys. 15, 075024 (2013).

[6] L. E. Kreilkamp, V. I. Belotelov, J. Y. Chin, S. Neutzner, D. Dregely, T. Wehlus, I. A. Akimov, M. Bayer, B. Stritzker, and H. Giessen, Phys. Rev. X 3, 041019 (2013).

[7] A. R. Davoyan, A. M. Mahmoud, and N. Engheta, Opt. Exp. 21, 3279 (2013).

[8] M. Moccia, G. Castaldi, V. Galdi, A. Alù, and N. Engheta, J. Phys. D: Appl. Phys. 47, 025002 (2014).
[9] M. Moccia, G. Castaldi, V. Galdi, A. Alù, and N. Engheta, J. Appl. Phys. 115, 043107 (2014).

[10] T. Kaihara, T. Ando, H. Shimizu, V. Zayets, H. Saito, K. Ando, and S. Yuasa, Opt. Exp. 23, 11537 (2015).

[11] R. Abdi-Ghaleh and R. Suldozi, Supperlat. Microstruct. 97, 242 (2016).

[12] X. Luo, M. Zhou, J. Liu, T. Qiu, and Z. Yu, Appl. Phys. Lett. 108, 131104 (2016).

[13] A. Chizhik, A. Zhukov, J. M. Blanco, and J. Gonzalez, J. Magn. Magn. Mater. 249, 27 (2002).

[14] A. A. Rzhevsky, B. B. Krichevtsov, D. E. Bürgler, and C. M. Schneider, Phys. Rev. B 75, 144416 (2007).

[15] K. Kämpf, S. Kübler, F. W. Herberg, and A. Ehresmann, J. Appl. Phys. 112, 034505 (2012).

[16] Y. Shoji, Y. Shirato, and T. Mizumoto, Jpn. J. Appl. Phys. 53, 022202 (2014).

[17] B. Caballero, A. García-Martín, and J. C. Cuevas, ACS Photon. 3, 203 (2016).

[18] A. V. Kimel, A. Kirilyuk, P. A. Usachev, R. V. Pisarev, A. M. Balbashov, and Th. Rasing, Nature (London) 435, 655 (2005). 
[19] K. Vahaplar, A. M. Kalashnikova, A. V. Kimel, D. Hinzke, U. Nowak, R. Chantrell, A. Tsukamoto, A. Itoh, A. Kirilyuk, and Th. Rasing, Phys. Rev. Lett. 103, 117201 (2009).

[20] A. Kirilyuk, A. V. Kimel, and Th. Rasing, Rev. Mod. Phys. 82, 2731 (2010).

[21] A. K. Zvezdin and V. A. Kotov, Modern Magnetooptics and Magnetooptical Materials, 1st ed. (Taylor and Francis, London, 1997).

[22] M. Inoue, M. Levy, and A. Baryshev, Magnetophotonics: From Theory to Applications, Springer Series in Material Sciences Vol. 178 (Springer-Verlag, Berlin Heidelberg, 2013), Chap. 4.

[23] D. Traviss, R. Bruck, B. Mills, M. Abb, and O. L. Muskens, Appl. Phys. Lett. 102, 121112 (2013).

[24] S. Feng and K. Halterman, Phys. Rev. B 86, 165103 (2012).

[25] J. Yoon, M. Zhou, Md. A. Bashda, T. Y. Kim, Y. C. Jun, and C. K. Hwangbo, Sci. Rep. 5, 12788 (2015).

[26] J. Park, J.-H. Kang, X. Liu, and M. L. Brongersma, Sci. Rep. 5, 15754 (2015).

[27] S. Vassant, A. Archambault, F. Marquier, F. Pardo, U. Gennser, A. Cavanna, J. L. Pelouard, and J. J. Greffet, Phys. Rev. Lett. 109, 237401 (2012).

[28] X. Sun, D. H. Kim, and H. S. Kwok, Materials Research Society Symposium Proceedings, Vol. 485, Thin-Film Structures for Photovoltaics, edited by E. D. Jones, J. Kalejs, R. Noufi, and B. Sopori (Cambridge University Press, New York, 1998), p. 267.

[29] I. A. Petukhov, A. N. Shatokhin, F. N. Putilin, M. N. Rumyantseva, V. F. Kozlovskii, A. M. Gaskov, D. A. Zuev, A. A. Lotin, O. A. Novodvorsky, and A. D. Khramova, Inorg. Mater. 48, 1020 (2012).

[30] S. Kang, S. Cho, and P. Song, Thin Solid Films 559, 92 (2014).

[31] H. Wang, H. Zhao, H. Su, G. Hu, and J. Zhang, Appl. Phys. Exp. 9, 092201 (2016).

[32] E. Feigenbaum, K. Diest, and H. A. Atwater, Nano Lett. 10, 2111 (2010).

[33] S. Q. Li, P. Guo, L. Zhang, W. Zhou, T. W. Odom, T. Seideman, J. B. Ketterson, and R. P. H. Chang, ACS Nano 5, 9161 (2011).

[34] N. P. K. Cotter, T. W. Preist, and J. R. Sambles, J. Opt. Soc. Am. A 12, 1097 (1995).
[35] D. M. Whittaker and I. S. Culshaw, Phys. Rev. B 60, 2610 (1999).

[36] B. Caballero, A. García-Martín, and J. C. Cuevas, Phys. Rev. B 85, 245103 (2012).

[37] C. A. Herreño-Fierro and E. J. Patiño, Phys. Stat. Sol. B 252, 316 (2015).

[38] J. B. González-Díaz, A. García-Martín, G. Armelles, J. M. García-Martín, C. Clavero, A. Cebollada, R. A. Lukaszew, J. R. Skuza, D. P. Kumah, and R. Clarke, Phys. Rev. B 76, 153402 (2007).

[39] Y. C. Jun, J. Reno, T. Ribaudo, E. Shaner, J.-J. Greffet, S. Vassant, F. Marquier, M. Sinclair, and I. Brener, Nano Lett. 13, 5391 (2013).

[40] A. Hiraki, E. Lugujjo, and J. W. Mayer, J. Appl. Phys. 43, 3643 (1972).

[41] T. Wehlus, T. Körner, S. Nowy, J. Frischeisen, H. Karl, B. Stritzker, and W. Brütting, Phys. Stat. Sol. A 208, 264 (2011).

[42] T. Gardner, C. Frisbie, and M. Wrighton, J. Am. Chem. Soc. 117, 6927 (1995).

[43] S. Oh, Y. Yun, D. Kim, and S. Han, Langmuir 15, 4690 (1999).

[44] S. K. VanderKam, E. S. Gawalt, J. Schwartz, and A. B. Bocarsly, Langmuir 15, 6598 (1999).

[45] C. Yan, M. Zharnikov, A. Gölzhäuser, and M. Grunze, Langmuir 16, 6208 (2000).

[46] P. M. Armistead and H. H. Thorp, Anal. Chem. 72, 3764 (2000).

[47] I. Markovich and D. Mandler, J. Electroanal. Chem. 500, 453 (2001).

[48] X. Yang, Y. Yuan, Z. Dai, F. Liu, and J. Huang, Sens. Actuat. B (Chem.) 237, 150 (2016).

[49] A. E. B. Costa, J. R. Mejía-Salazar, and S. B. Cavalcanti, J. Opt. Soc. Am. B 33, 468 (2016).

[50] D. J. Bergman, Phys. Rep. 43, 377 (1978).

[51] M. Born and E. Wolf, Principles of Optics (Pergamon, New York, 1980).

[52] V. M. Agranovich and V. E. Kravtsov, Solid State Commun. 55, 85 (1985).

[53] P. A. Belov and Y. Hao, Phys. Rev. B 73, 113110 (2006).

[54] P. B. Catrysse and S. Fan, Phys. Rev. Lett. 106, 223902 (2011). 